\title{
PERSPECTIVE
}

\section{Management of ocular ischaemic syndrome}

\author{
Raman Malhotra, Kevin Gregory-Evans
}

Symptoms of carotid artery disease frequently present to ophthalmologists. Though these may be sight threatening, they may represent the first signs of life threatening carotid artery stenosis. These include cerebral transient ischaemic attacks (TIA), transient monocular blindness (amaurosis fugax), central or branch retinal artery occlusion, hypotensive retinopathy (previously known as "venous stasis retinopathy"), and ocular ischaemic syndrome (OIS). ${ }^{1}$

Of these associations with carotid artery disease, OIS presents the most challenging condition for the ophthalmologist with many controversial aspects to its management.

OIS is a severe form of chronic ischaemia of both anterior and posterior segments of the eye as well other orbital structures supplied by the ophthalmic artery. It is thought to be due to chronic hypoperfusion when carotid artery stenosis is greater than $90 \%{ }^{1}$

OIS is rare; however, ophthalmoscopic features of hypotensive retinopathy have been found in $5 \%-20 \%{ }^{2}$ of patients with carotid artery occlusive disease and approximately 200 patients with OIS have been reported in the literature by way of case reports, retrospective reviews, and prospective studies. ${ }^{3}$ Based on a questionnaire survey it has been estimated that a neuro-ophthalmologist or glaucoma specialist encounters at least one case per year. ${ }^{4}$

In this article we present an overview and recommendations for best clinical practice for OIS based on an extensive review of recent studies.

\section{Clinical findings in OIS}

SYMPTOMS

Clinical presentation may include sudden (41\%), gradual $(28 \%)$, or transient vision loss $(15 \%)$ or pain, either ocular or orbital $(13 \%)$. In $20 \%$ of cases, the clinical signs of OIS are an incidental, asymptomatic finding. ${ }^{3}$ Brown et $a l,{ }^{5}$ in their retrospective study of 43 patients, showed that $90 \%$ had reduced visual acuity and in two thirds of patients this occurred gradually over a period of weeks to months.

Rarely, vision loss may be precipitated by exposure to bright lights ("bright light amaurosis") with subjective after-images of visual distortion, fragmentation, dazzle, or just blurring. It may also occur with change in posture or even exertion. ${ }^{6-8}$ It is likely that this phenomenon represents photoreceptor ischaemia due to poor retinal and choroidal circulation. Russell and Ikeda ${ }^{8}$ showed that patients with carotid artery stenosis whose main symptom was unilateral bright light amaurosis had marked ERG delay in the recovery time of b-wave amplitude after photostress. These patients also had associated fluorescein fundus angiogram (FFA) changes of patchy choroidal filling.

Ocular discomfort or pain around the orbit in OIS, in the absence of glaucoma, occurs in $5-10 \%$ of patients. ${ }^{59}$ It often reduces on lying down and is thought to be due to ischaemic damage to the branches of the ophthalmic division of the trigeminal nerve. ${ }^{6} 10$

SIGNS

Anterior segment signs include dilated episcleral vessels, corneal oedema, anterior chamber cells, and pronounced flare ("ischaemic pseudo-inflammatory uveitis"), a middilated poorly reactive pupil, cataract, iris atrophy, iris neovascularisation with or without angle neovascularisation, or neovascular glaucoma. ${ }^{1610}$

Iris neovascularisation has been found in up to $90 \%$ of cases, and dilated episcleral vessels and uveitis in up to $20 \%$ of cases. $^{35}$

Diffuse episcleral injection, as opposed to the ciliary flush of uveitis, may be due to increased collateral blood flow in the presence of internal carotid artery (ICA) occlusion. Countee $e t a l^{11}$ reported seven patients with episcleral injection and ipsilateral ICA occlusion, with no evidence of OIS, in whom arteriography confirmed dilated external carotid artery collateral vessels in the orbit supplying an enlarged ophthalmic artery by retrograde flow. The authors concluded that dilated episcleral arteries may be an important physical finding in patients suspected of having an ipsilateral ICA occlusion, and may suggest that the external carotid artery is the major source of blood supply to the ipsilateral cerebral hemisphere.

Iris neovascularisation seen at presentation is considered an indicator of poor visual prognosis. Over $95 \%$ of such eyes develop a visual acuity of counting fingers or less within 1 year. ${ }^{12}{ }^{13}$ Intraocular pressure (IOP) is usually raised. However, it may be normal or even reduced, despite iris neovascularisation, presumably due to ciliary body ischaemia leading to reduced aqueous production. Two thirds of patients will have an IOP of $<22 \mathrm{~mm} \mathrm{Hg}$ at presentation. $^{35}$

Posterior segment signs include venous dilatation with or without tortuosity, mid-peripheral retinal haemorrhages and microaneurysms, and an easily induced retinal artery pulsation with gentle digital pressure. Ischaemic changes include retinal arteriolar narrowing, retinal capillary non-perfusion, macular oedema, optic disc neovascularisation (NVD) and, less commonly, retinal neovascularisation (NVE). ${ }^{13561415}$ Easily inducible or even spontaneous retinal artery pulsation is present in most cases. It is a striking feature and most pronounced near the optic disc. ${ }^{5}$

Severe carotid artery stenosis has also been suggested to explain asymmetry in proliferative diabetic retinopathy. ${ }^{16-18}$ In diabetic patients with OIS, in whom the anterior segment signs are mild or even absent, it may appear, therefore, as asymmetrical proliferative diabetic retinopathy.

Anterior ischaemic optic neuropathy has been reported in association with OIS as a rare complication of carotid artery obstruction. It is believed that this is due to an inadequate perfusion pressure within the deep capillaries of the optic nerve head. ${ }^{19}$

DIFFERENTIAL DIAGNOSIS

It is important to exclude other important causes of iris neovascularisation such as proliferative diabetic retin- 
opathy and ischaemic central retinal vein occlusion (CRVO). Retinal arterial pressure should be normal in eyes with diabetic retinopathy and venous occlusion.

\section{INVESTIGATIONS}

Ocular

Fluorescein fundus angiography-The fluorescein angiographic signs of OIS include delayed and patchy choroidal filling, increased retinal arteriovenous circulation times, areas of retinal capillary non-perfusion, late leakage from arterioles and veins, leakage from new vessels, and macular oedema. ${ }^{13520}$

Leakage from retinal vessels has been reported to be present in $85 \%$ of eyes with OIS, presumably due to ischaemic endothelial cell dysfunction. In combination with leakage from microaneurysms this appears to account for macular oedema when present. ${ }^{5}$

With this in mind, during angiographic evaluation of macular oedema in routine practice, any delay in filling time should raise the possibility of coincidental carotid artery disease.

Retinal capillary non-perfusion is sometimes present in OIS. Brown and Magargal ${ }^{5}$ reviewed the FFA of 40 eyes of 40 patients and found retinal capillary non-perfusion in some cases, most often in the mid-periphery with a gradual transition. The authors did not specify any further details as to the frequency or extent of this finding. This contrasts with the sharply demarcated border between areas of retinal capillary perfusion and non-perfusion that are seen with, for example, an ischaemic central vein occlusion. However, Mizener et $a l^{3}$ failed to show any retinal capillary non-perfusion, even diabetics, in 22 eyes from 15 patients suitable for FFA. The authors emphasise the point that in the absence of capillary non-perfusion the results of diabetic trials for proliferative diabetic retinopathy and therefore the beneficial value of pan-retinal laser photocoagulation (PRP) $)^{21}$ may not be applicable to OIS.

The role of FFA in OIS is therefore to aid confirmation of diagnosis, determine the cause of iris neovascularisation, and to demonstrate retinal capillary non-perfusion in order to validate the indication for PRP.

Visual fields-Patients with OIS have been found on presentation to have visual fields that vary from normal (23\%) to central scotomas (27\%), nasal defects (23\%), centrocaecal defects $(5 \%)$, and only central or temporal islands present $(22 \%)$.

Orbital ophthalmic colour Doppler ultrasound - Colour Doppler imaging (CDI) can be useful. This is a recent advance in ultrasonography that provides colour coded blood flow data of vessels at the same time as conventional real time grey scale B-scan images. Retrobulbar colour Doppler ultrasound findings in OIS with more than $70 \%$ carotid artery stenosis include reduced peak systolic velocities in ophthalmic and central retinal arteries and continuous or intermittent reversal of ophthalmic artery blood flow. ${ }^{22-25}$ However, colour Doppler imaging has limited clinical use at present because measurements of flow velocity in orbital vessels are poorly reproduced, the most reliable being those of the ophthalmic artery nasal to the globe. ${ }^{26}$

\section{General}

Systemic diseases most often associated with OIS include diabetes mellitus (56\%), hypertension (50-73\%), ischaemic heart disease (38-48\%), and cerebrovascular disease $(27-31 \%) .^{327}$ The prevalence of hypertension and ischaemic heart disease was comparable with those found in patients with retinal embolic disease. However, the prevalence of diabetes mellitus in OIS was not only greater than the aged matched population but also greater than in patients with retinal embolic disease.$^{27}$ It is suggested that perhaps the combination of both large vessel and small vessel disease increases the risk of OIS.

Importantly, the 5 year mortality rate in OIS patients is as high as $40 \%$. $^{27}$ The majority of deaths are due to cardiac disease.

\section{Management of OIS}

Controversy in the management of OIS arises from the fact that the majority of patients reported in the literature are part of small retrospective series or case reports. Only one prospective study ${ }^{3}$ with 39 eyes of 32 patients has been published and the largest retrospective series ${ }^{12} 27$ collected only 52 cases over 8 years in an outpatient clinic that recorded 1.5 million visits during that period. With such a rare disease, it is very difficult to carry out a randomised prospective trial evaluating the effects of treatment on visual outcome.

ROLE OF THE OPHTHALMOLOGIST

Ocular treatment is directed towards the treatment of anterior segment inflammation, ablation of retinal ischaemia (if present), and the control of raised intraocular pressure and neovascular glaucoma (NVG).

It is important to diagnose OIS early. Intraocular pressure needs to be controlled and attention directed towards the prevention of NVG. To this purpose hypotensive retinopathy should be assessed and monitored both clinically and with FFA in order to identify retinal ischaemia and validate the need for effective laser PRP. In the acute stage it is important to arrange FFA as soon as possible before significant corneal oedema precludes a view for funduscopy and effective laser PRP.

Anterior segment inflammation may be treated with regular topical steroid and cycloplegics. Topical $\beta$ adrenergic antagonists or $\alpha$ adrenergic agonists along with oral carbonic anhydrase inhibitors are first line therapy for raised IOP; however, they may only reduce IOP temporarily. Topical cycloplegia along with oral analgesics are required for pain relief.

Optic nerve function monitoring, including visual fields, is important as a guide to the progression of disease, the effect of treatment, and the presence of coincidental, treatable ocular disease.

It should not be forgotten that patients with bilateral disease will often be eligible for registration as blind or partially sighted by way of field loss before central vision is dramatically affected. Patients with reduced visual acuity will require low vision aids.

A prompt referral for full medical and neurological assessment in order to optimise systemic risk factors and associated cardiovascular disease is needed. Medical treatment would include aspirin or another antiplatelet drug, treatment of hypertension and diabetes, and advice to stop smoking. ${ }^{28}$ The decision regarding treatment for carotid artery disease requires both the neurologist and vascular surgeon.

ROLE OF PANRETINAL PHOTOCOAGULATION

Panretinal photocoagulation (PRP) is the accepted treatment for retinal ischaemia predisposing to neovascularisation in diabetic eye disease. ${ }^{21}$ It is thought that retinal ischaemia triggers the production of retinal angiogenic growth factors that stimulate retinal (NVE) and optic nerve head (NVD) new vessel growth and possibly diffuse into the anterior segment giving rise to iris neovascularisation (NVI). ${ }^{29}{ }^{30}$ By ablating ischaemic retina, it is thought that PRP reduces the production of growth factors thereby leading to regression of neovascularisation thus preventing NVG. 
In OIS, the occurrence of NVI has traditionally been attributed to severe retinal ischaemia. It has been found, however, that PRP alone causes NVI regression in only $36 \%$ of eyes. ${ }^{12}$ Laser PRP is thought not to be as effective in reducing the ischaemic stimulus for NVI as for diabetic neovascularisation. ${ }^{4}$ In this context it is notable that Mizener $e t a l^{3}$ found no evidence of retinal ischaemia, in the form of capillary dropout on angiography in OIS patients, even in those with coincidental diabetes mellitus. In animal studies Hayreh and Baines ${ }^{31}$ experimentally induced NVI due to uveal ischaemia in rhesus monkeys without any retinal ischaemia. It has been suggested therefore, that uveal ischaemia alone may be responsible for neovascularisation in some cases of OIS.

These observations highlight the importance of angiography in the investigation of OIS. It has been suggested that PRP in OIS should be reserved for cases of established retinal ischaemia. In this context full peripheral retinal ablation $3000-5000$ burns of $200-500 \mu \mathrm{m}$ spot size be used. Hayreh ${ }^{32}$ comments that there is no scientific rationale for PRP when FFA shows no retinal ischaemia in the form of capillary non-perfusion. In such cases of choroidal or ciliary body ischaemia rather than retinal ischaemia, the complications of PRP such as pain and further visual field constriction are therefore avoided.

\section{CONTROL OF INTRAOCULAR PRESSURE}

NVG is notoriously difficult to treat in cases of OIS. Chronic IOP elevation in the presence of compromised ocular perfusion can lead to anterior ischaemic optic neuropathy, central retinal artery occlusion, corneal oedema, and a blind painful eye.

Topical $\beta$ adrenergic antagonists or $\alpha$ adrenergic agonists, topical steroids, and cycloplegics along with oral carbonic anhydrase inhibitors may temporarily help in reducing IOP and inflammation. However, medical therapy is usually not effective in controlling IOP in the intermediate to long term because the trabecular meshwork is physically occluded with neovascular tissue and fibrosis. Attention should therefore also be directed towards eliminating any ischaemic retina present. Conventional filtering surgery (trabeculectomy usually with mitomycin C) also carries a limited chance of success in the presence of NVI. Rarely, tube shunt procedures (such as the Molteno tube or Ahmed valve implant) may be considered as a primary procedure or after failed conventional filtering surgery in a sighted eye. ${ }^{33}$

Ciliary body ablation, however, has been shown to be effective in controlling IOP in end stage refractory glaucomas such as NVG. Methods such as cyclocryotherapy and laser cyclophotocoagulation are well described. Until recently the $1064 \mathrm{~nm}$ contact or non-contact neodymium:YAG (Nd:YAG) laser has been a popular modality. More recently, however, the $810 \mathrm{~nm}$ semiconductor diode laser has been shown to be better absorbed by ciliary body pigment and offers more effective cycloablation. ${ }^{34}$

Diode laser (cyclodiode) ciliary ablation is reported to cause less inflammation than Nd:YAG laser, is less painful, and results in a more predictable final IOP. Complications such as phthisis bulbi, hypotony, and uveitis are also considered to be less frequent. ${ }^{35}{ }^{36}$ A typical protocol strategy would be to apply approximately 10 laser burns of 1500-2000 $\mathrm{mW}$ for $1500-2000 \mathrm{~ms}$ in each quadrant. To titrate treatment with response, these laser applications may be applied over a number of treatment sessions. ${ }^{35-37} \mathrm{An}$ appropriate end point would be long term control of intraocular pressure with clearing of corneal oedema. There may or may not be concomitant improvement in visual function. Clinical regression of NVI using peripheral trans-scleral retinal diode combined with cyclodiode is also reported..$^{38}$

ROLE OF CAROTID SURGERY

OIS is usually an important indicator of carotid artery stenosis, and all OIS patients should be referred for neurological and cardiovascular assessment at the time of ocular diagnosis. Carotid endarterectomy has been shown to benefit patients with symptomatic cerebral ischaemia when there is greater than $70 \%$ carotid artery stenosis. ${ }^{28}$

Based on retrobulbar colour Doppler ultrasound examinations, carotid endarterectomy has been shown to improve ocular blood flow. Peak systolic velocity of flow in the ophthalmic artery rises after surgery ${ }^{25} 3940$ and any reversal of ophthalmic artery flow is corrected..$^{40}$ Carotid artery surgery therefore can reduce ocular ischaemia and improve hypotensive retinopathy as well as reduce the risk of stroke.

Although reports exist of IOP rising as ciliary body circulation is improved by carotid endarterectomy or by superficial temporal artery-middle cerebral artery bypass surgery (STA-MCA), ${ }^{91}$ most patients undergoing carotid endarterectomy do not experience any significant rise in IOP. $^{312}$

Clinical signs of hypotensive retinopathy have been reported to regress following carotid surgery. ${ }^{9}{ }^{1342-44}$ FFA changes following surgery include reduction in arteriovenous transit time, macular oedema, and microaneurysms present. $^{44}$

Surgery has also been shown in selected cases to help regression of iris neovascularisation ${ }^{12} 144^{45}$ and neovascular glaucoma. ${ }^{45} 46$

It should be borne in mind that many of the cases reported above were of patients undergoing STA-MCA bypass surgery rather than carotid endarterectomy. Bypass surgery has been advocated if lesions are unresectable by carotid endarterectomy. Such a situation would include cases of total internal carotid stenosis, common carotid occlusion, or diffuse ulcerative stenosis extending distally along the internal carotid artery. ${ }^{12}$ More recently, an international randomised clinical trial of STA-MCA bypass surgery in patients with symptomatic carotid occlusive disease failed to show any protection against cerebral ischaemia with no reduction in stroke rate. ${ }^{47}$ STA-MCA bypass surgery is no longer a widely accepted alternative to carotid endarterectomy and, to some extent, the case for carotid artery surgery in OIS is still not proved. The European Carotid Surgery Trial (ECST) results showed that the risk of ischaemic stoke over 3 years in symptomatic patients with $70-99 \%$ carotid stenosis on medical treatment alone was only about $20 \%{ }^{28}$ They also showed that carotid endarterectomy lowered this risk by $50 \%$ over 3 years. Therefore, surgery had no benefit and possibly harmful effects in the remaining $80 \%$ of patients. ${ }^{48} \mathrm{~A}$ recent prognostic risk model based on the ECST proposed a "risk factor score" and suggested that patients with severe carotid stenosis and a recent cerebral rather than ocular event had a greater risk of stroke when taking medical treatment and therefore a greater benefit from surgery. ${ }^{49}$

It should also be remembered that carotid surgery may not alter long term visual outcome in the affected eye. ${ }^{312} 13$ Cases of early improved visual acuity after surgery have been reported, ${ }^{21}$ and a small retrospective series reported stabilisation of visual acuity ${ }^{45}$; however the authors did not report long term visual outcome, for example, at 1 year.

\section{Conclusion}

OIS is a severe but rare condition, often leading to significant visual loss and chronic ocular pain. Iris neovascularisation is an indicator of poor visual prognosis. Diagnosis 
should aim to detect early disease before the onset of iris neovascularisation. Current medical and laser treatment protocols can successfully control progression of disease although prognosis for vision is usually poor and it is difficult to make clear recommendations for aggressive therapy. It is important to recognise that the carotid circulation is significantly compromised in most cases of OIS and if life threatening neurological or cardiovascular complications are to be avoided, prompt neurological and medical assessment should be sought.

RAMAN MALHOTRA

The Oxford Eye Hospital, Radcliffe Infirmary, Walton Street, Oxford OX2 6HE, UK

malhotraraman@hotmail.com

KEVIN GREGORY-EVANS

The Western Eye Hospital, Marylebone Road, London NW1 5YE,

UK

k.gregory-evans@ic.ac.uk

1 Dugan JD Jr, Green WR. Ophthalmologic manifestations of carotid occlusive disease.Eye 1991;5(Pt 2):226-38.

2 Kiser WD, Gonder J, Magargal LE, et al. Recovery of vision following treatment of the ocular ischemic syndrome. Ann Ophthalmol 1983.;15:305.

3 Mizener JB, Podhajsky P, Hayreh SS. Ocular ischemic syndrome.Ophthalmology 1997; 104:859-64.

4 Mills RP. Anterior segment ischemia secondary to carotid occlusive disease. 7 Clin Neuro-ophthalmol 1989;9:200-4.

5 Brown GC, Magargal LE. The ocular ischaemic syndrome. Clinical, fluorescein angiographic and carotid angiographic features. Int Ophthalmol 1988;11:239

6 Jacobs NA, Ridgway AEA. Syndrome of ischaemic ocular inflammation: six cases and a review. Br f Ophthalmol 1985;69:681

7 Donnan GA, Sharbrough FW, Whisnant JP. Carotid occlusive disease. Effect of bright light on visual evoked response. Arch Neurol 1982;39:6879.

8 Russell RW, Ikeda H. Clinical and electrophysiological observations in patients with low pressure retinopathy. Br f Ophthalmol 1986;70:651-6.

9 Kearns TP, Siekert RG, Sundt TM. The ocular aspects of bypass surgery of the carotid artery. Mayo Clin Proc 54;3:1979.

10 Knox DL. Ischaemic ocular inflammation. Am f Ophthalmol 1965;60:995.

11 Countee RW, Gnanadev A, Chavis P. Dilated episcleral arteries-a significant physical finding in assessment of patients with cerebrovascular insufficiency. Stroke 1978;9:42-5.

12 Sivalingam A, Brown GC, Magargal LE. The ocular ischaemic syndrome. Visual prognosis and the effect of treatment. Int Ophthalmol 1991;15:1520.

13 Ino-ue M, Azumi A, Kajiura-Tsukahara Y, et al. Ocular ischemic syndrome in diabetic patients. Fpn $\mathcal{F}$ Ophthalmol 1999;43:31-5.

14 Sturrock GD, Mueller HR. Chronic ocular ischaemia. $\mathrm{Br} f$ Ophthalmol 1984;68:716-23.

15 Ros MA, Margargal LE, Hedges Jr TR, et al. Ocular ischaemia: long term complications. Ann Ophthalmol 1987;19:270-2

16 Dogru $M$, Ino-Ue $M$, Nakamura $M$, et al. Modifying factors related to asymmetric diabetic retinopathy. Eye 1998;12:929-33.

7 Duker JS, Brown GC, Bosley TM, et al. Asymmetric proliferative diabetic retinopathy and carotid artery disease. Ophthalmology 1990;97:869-74.

18 Moss SE, Klein R, Klein BE. Ocular factors in the incidence and progression of diabetic retinopathy. Ophthalmology 1994;101:77-83.

19 Brown GC. Anterior ischemic optic neuropathy occuring in association with carotid artery obstruction. F Clin Neuro-ophthalmol 1986;6:29-42.

20 Sarkies NJC, Shilling JS, Ross Russell RW. Fluorescein angiography in carotid disease. Trans Opthalmol Soc UK 1986;105:489-93.

21 Diabetic Retinopathy Study Research Group. Photocoagulation treatment of proliferative diabetic retinopathy:clinical application of diabetic retin-
opathy study (DRS) findings. DRS report No 8. Ophthalmology opathy study (D)
$1981 ; 88: 583-600$
22 Costa VP, Kuzniec S, Molnar LJ, et al. Clinical findings and hemodynamic changes associated with severe occlusive carotid artery disease. Ophthalmology 1997;104:1994-2002.

23 Riordan-Eva P, Restori M, Hamilton AM, et al. Orbital ultrasound in ocular ischaemic syndrome. Eye 1994;8:93-6.

24 Lee HM, Fu ER. Orbital colour Doppler imaging in chronic ocular ischaemic syndrome. Aust NZ f Ophthalmol. 1997;25:157-63.

25 Mawn LA, Hedges TR 3rd, Rand W, et al. Orbital color Doppler imaging in carotid occlusive disease. Arch Ophthalmol 1997;115:492-6.

26 Williams TH, Harris A. Colour Doppler ultrasound imaging of the eye and orbit. Surv Ophthalmol 1996;40:255-67.

27 Sivalingam A, Brown GC, Magargal LE. The ocular ischaemic syndrome. Mortality and systemic morbidity. Int Ophthalmol 1989;13:187-91.

28 European Carotid Surgery Trialists Collaborative Group. MRC European Carotid Surgery Trial: interim results for symptomatic patients with severe (70-99\%) or with mild (0-29\%) carotid stenosis. Lancet 1991;337:123543.

29 Dodson PM, Gibson JM, Kritzinger EE. Clinical retinopathies. London: Chapman and Hall, 1995

30 Matthews MK, Merges C, McLeod DS, et al. Vascular endothelial growth factor and vascular permeability changes in human diabetic retinopathy. Invest Ophthalmol Vis Sci 1997;38:2729-41.

31 Hayreh SS, Baines JAB. Occlusion of the vortex veins. An experimental study. Br F Ophthalmol 1973;57:217.

32 Hayreh SS. Chronic ocular ischemic syndrome in internal carotid artery occlusive disease: controversy on "venous stasis retinopathy". In: Bernstein EF, ed. Amaurosis fugax. New York: Springer-Verlag 1988:135-58.

33 Ritch R, Shields B, Krupin T. The glaucomas. 2nd ed. Vol 2. St Louis: Mosby, 1996

34 Assia EI, Hennis HL, Stewart WC, et al. A comparison of neodymium: yttrium aluminium garnet and diode laser trans-scleral cyclophotocoagulation and cyclocryotherapy. Invest Ophthalmol Vis Sci 1991;32:2774-8.

35 Spencer AF, Vernon SA. "Cyclodiode": results of a standard protocol. $\mathrm{Br} \mathcal{F}$ Ophthalmol 1999;83:311-16.

36 Bloom PA, Tsai JC, Sharma K, et al. Cyclodiode: trans-scleral diode laser cyclophotocoagulation in the treatment of advanced refractory glaucoma. Ophthalmology 1997;104:1508-19.

37 Walland MJ. Diode laser cyclophotocoagulation: dose-standardized therapy in end-stage glaucoma. Aust NZ F Ophthalmol 1998;26:135-9.

38 Flaxel CJ, Larkin GB, Broadway DB, et al. Peripheral transscleral retinal diode laser for rubeosis iridis. Retina 1997;17:421-9.

39 Wong YM, Clark JB, Faris IB, et al. The effects of carotid endarterectomy on ocular haemodynamics. Eye 1998;12:367-73.

40 Costa VP, Kuzniec S, Molnar LJ, et al. The effects of carotid endarterectomy on the retrobulbar circulation of patients with severe occlusive carotid artery disease. Ophthalmology 1999;106:306-10

41 Young LH, Appen RE. Ischemic oculopathy. A manifestation of carotid artery disease. Arch Neurol 1981;38:358-61.

42 Neupert JR, Brubaker RF, Kearns TP, et al. Rapid resolution of venous stasis retinopathy after carotid endarterectomy. Am f Ophthalmol 1976;81: 600-2.

43 Edwards MS, Chater NL, Stanley JA. Reversal of chronic ocular ischaemia by extracranial-intracranial arterial by-pass: case report. Neurosurgery 1980;7:480-3

44 Sarkies NJC, Shilling JS, Burnard KG, et al. Assessment by fluorescein angiography of surgical treatment of occlusive carotid artery disease. Stroke 1987;18:585-90.

45 Johnston ME, Gonder JR, Canny CL. Successful treatment of ocular ischaemic syndrome with panretinal photocoagulation and cerebrovacular surgery. Can f Ophthalmol 1988;23:114-19.

46 Kearns TP, Seibert RG. The ocular aspects of carotid artery surgery. Trans Am Ophthalmol Soc 1978;76:247-65.

47 The EC/IC Bypass Study Group. Failure if extracranial-intracranial arterial bypass to reduce the risk of ischemic stroke: results of an international randomized trial. N Engl f Med 1985;313:1191-211.

48 Rothwell PM, Slattery J, Warlow CP. A systematic review of the risks of stroke and death due to carotid endarterectomy for symptomatic stenosis. Stroke 1996;27:260-5.

49 Rothwell PM, Warlow CP. Prediction of benefit from carotid endarterectomy in individual patients: a risk-modelling study. European Carotid Surgery Trialists' Collaborative Group. Lancet 1999;353:2105-10. 